\title{
Biotin as a reactive handle to selectively label proteins and DNA with small molecules
}

\author{
Adam D. Cotton ${ }^{1}$, James A. Wells ${ }^{1}$, and Ian B. Seiple ${ }^{1,2}$ \\ 1 - Department of Pharmaceutical Chemistry, University of California, San Francisco, 94143, USA \\ 2 - Cardiovascular Research Institute, University of California, San Francisco, 94143, USA \\ \# - Correspondence to ian.seiple@ucsf.edu
}

\begin{abstract}
Biotin labeling of proteins and DNA is probably the most common bioconjugation for affinity immobilization, but has not been exploited for chemical modification. Recently, oxaziridine reagents were developed that selectively conjugate the thioether of methionines on the surface of proteins, a method termed redox-activated chemical tagging (ReACT). These reagents generate sulfimide linkages that range in stability depending on solvent accessibility and substitutions on the oxaziridine. Here we show that oxaziridine reagents react rapidly with the thioether in biotin to produce sulfimide products that are stable for more than seven days at $37^{\circ} \mathrm{C}$. This method, which we call biotin redox-activated chemical tagging (BioReACT) expands the utility of biotin labeling and enables predictable and stable chemical conjugation to biomolecules without the need to screen for a suitable methionine conjugation sites. We demonstrate the versatility of this approach by producing a fluorescently labeled antibody, an antibodydrug conjugate, and a small molecule-conjugated oligonucleotide. We anticipate that BioReACT will be useful to researchers to rapidly introduce biorthogonal handles into biomolecules using biotin, a functional group that is widespread and straightforward to install.
\end{abstract}

Chemical modification of biomolecules has widespread applications ranging from antibody-drug conjugates to in vivo localization assays. ${ }^{1,2,3}$ Researchers have devised numerous methods to conjugate small molecules to biomolecules such as amino acid modification or introduction of a biorthogonal reactive handle. ${ }^{4}$ Due to their prevalence and reactivity, lysine and cysteine are the most widely used amino acids. While methionine has been the focus of other labelling strategies ${ }^{5}$, its relatively unreactive side chain makes bioconjugation challenging. Recently, the Chang and Toste groups described oxaziridine chemistry for selective methionine modification on proteins called redox-activated chemical tagging (ReACT). ${ }^{6}$ Methionine is seldom found on the surface of proteins, enabling accurate and site-specific labelling ${ }^{7,8}$. Although the ReACT method is extremely selective, the conjugation product is prone to hydrolysis, especially at the when the label is installed at a C-terminal methionine, limiting its potential for applications where stability is critical. ${ }^{9}$ To address this limitation, more stable oxaziridine reagents were synthesized and our group identified sites on the general antibody scaffold that both labels well and are stable for several days. ${ }^{9,10}$ The most stable sites to hydrolysis were in partially buried regions of the antibody that were not entirely predictable. A method to generate oxaziridine-biomolecule linkages at accessible sites with predictable stability would be advantageous.

Biotin is ubiquitous throughout biological research, and proteins and DNA can usually be biotinylated with no effect on function. It is regularly used in conjunction with avidin-based reagents, enabling applications such as immobilization or fluorescent tagging. ${ }^{11,12,13,14,15}$ Avidin is a tetrameric protein that can introduce unwanted avidity effects and additional protein complications to numerous biophysical assays. Furthermore, unless the it is pre-modified, avidin cannot be used to introduce small molecule conjugates. Oxaziridine reagents such as those used in ReACT are susceptible to nucleophilic attack by methionine into the $\mathrm{N}-\mathrm{O} \sigma^{*}$ followed by elimination resulting in a sulfimidated methionine (Figure 1a). We hypothesized that oxaziridine reagents might also react with the tetrahydrothiophene in biotin, ${ }^{16}$ enabling rapid small molecule bioconjugation to proteins and other biomolecules. In addition, we hypothesized that the increased steric hinderance of the biotin bicycle would alter the reactivity and potentially decrease the hydrolysis rate of the resulting sulfimide conjugate.

Here we report the reaction between biotin and azide-labelled oxaziridine reagents in aqueous conditions at room temperature. This method, which we call biotin redox-activated chemical tagging (BioReACT), achieves efficient and stable labelling of proteins with oxaziridine reagents. We functionally validate the method by generating an antibody-drug conjugate and numerous flow-cytometry reagents. Finally, we conjugate a functional click handle to a biotinylated oligonucleotide. These studies show that the biotin-oxaziridine reaction is a powerful approach for the efficient synthesis of stable protein and DNA bioconjugates.

We first evaluated the reactivity of unconjugated biotin with oxaziridine reagents by exposing biotin methyl ester 1 to oxaziridine 2 (prepared from known protocol) in $1: 1 \mathrm{CD}_{3} \mathrm{OD} / \mathrm{D}_{2} \mathrm{O}$ (Fig. 1b). ${ }^{9}$ The two protons alpha to the sulfur in biotin appear as distinct peaks between 2.7-3.0 ppm in ${ }^{1} \mathrm{H}$ NMR, allowing us to assess the conversion and reaction kinetics. Remarkably, the reaction proceeds to $>80 \%$ conversion within 3 minutes, and $100 \%$ conversion by 20 minutes (Fig. 1c, S1). We observed a 17:3 ratio of N-transfer product (NTP, sulfimide) to O-transfer product (OTP, sulfoxide), which proved challenging to separate, resulting in an $85 \%$ NMR yield and 52\% isolated yield of 
desired sulfimide 3 . It should be noted that while we observed OTP in this reaction, it was never seen for any of the biologically labeling experiments described below.

a<smiles>CCCCCCCCCCCCCCCCCSC</smiles>

Previous work (Met Labelling)

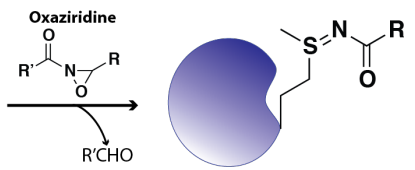

b

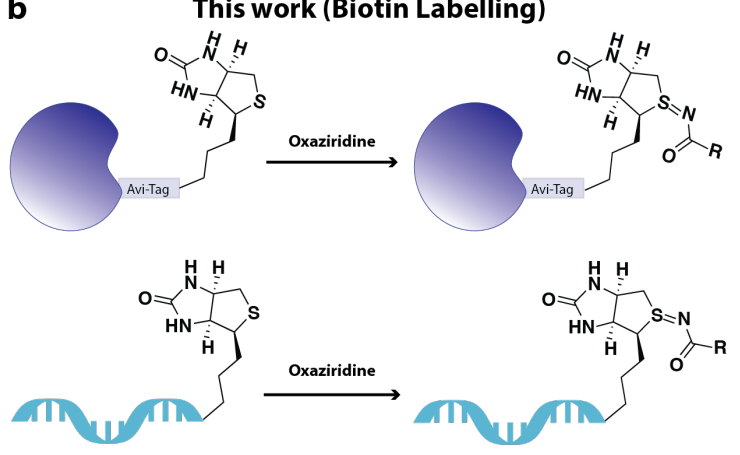

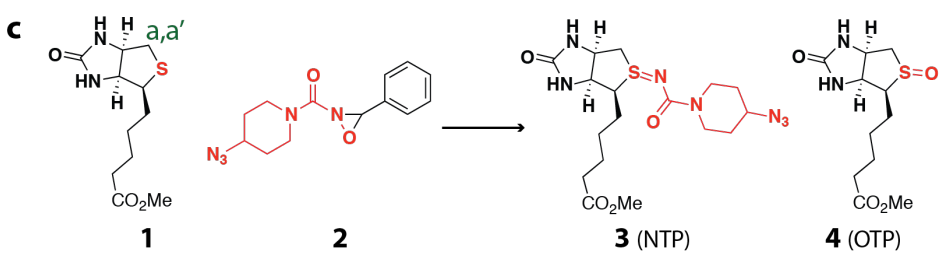

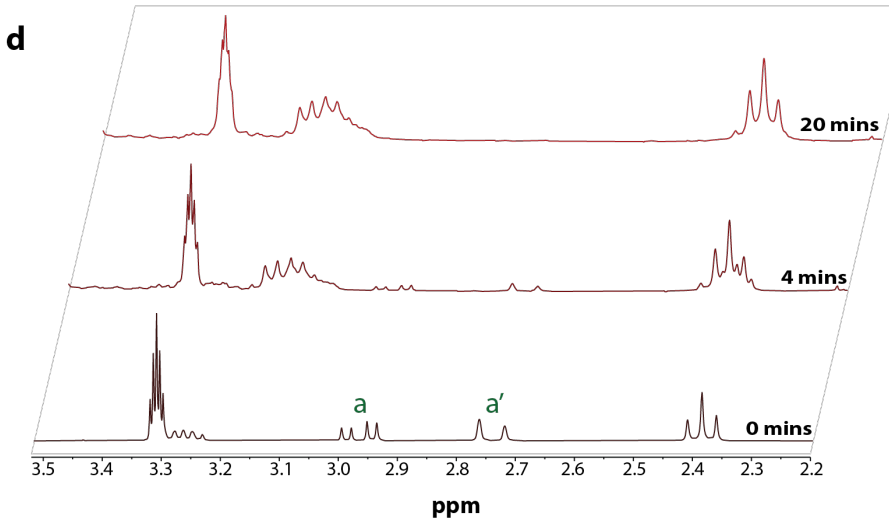

Figure 1: Repurposing oxaziridines to label biotin. a) ReACT strategy for methionine bioconjugation. Oxaziridine compounds react with the thioether in methionine to form a sulfimide conjugation product. b) Hypothesized reactivity of oxaziridines with biotin to form a sulfimidated bicycle. BioReACT enables labeling of biomolecules such as DNA that contain no methionines. c) The reaction of biotin methyl ester with 1.5 equivalents of oxaziridine 2 in $1: 1 \mathrm{CD}_{3} \mathrm{OD} / \mathrm{D}_{2} \mathrm{O}$ to form the resulting NTP sulfimide (85\% NMR yield, $52 \%$ isolated yield). d) $1 \mathrm{H}-\mathrm{NMR}$ series of the reaction in (c). Conversion was monitored by detecting the chemical shift of the protons alpha to the sulfur at $\sim 2.75$ and $\sim 2.97 \mathrm{ppm}$. Conversion was complete within 20 minutes.

We next evaluated the reactivity of biotin attached to antigenbinding fragment (Fab) derived from the anti-Her2 antibody trastuzumab as a model protein. The Fab scaffold has been thoroughly characterized with the methionine strategy (ReACT) to identify reactive and stable sites, making it a privileged candidate for comparison of the two labelling strategies. ${ }^{8}$ We chose trastuzumab as it expresses well and has high thermal stability. We incubated $50 \mu \mathrm{M}$ biotinylated trastuzumab Fab in PBS at $23{ }^{\circ} \mathrm{C}$ with a several concentrations of oxaziridine 2 and used whole protein mass spectrometry to measure labelling. Gratifyingly, we saw labelling at equimolar concentrations of Fab and oxaziridine, and quantitative labelled protein at 10-fold excess oxaziridine (Fig. S2). WT unbiotinylated trastuzumab Fab contains three native methionines ${ }^{17}$ that were minimally reactive to the oxaziridine reagent (Fig. 2a). At high molar excess 2, we saw increased labelling of the native protein, thus for future experiments we used 5-fold molar excess oxaziridine reagent. To gauge the working concentration range of labelling we incubated trastuzumab Fab at increasing concentrations with 5-fold molar excess of oxaziridine probe (Fig. S3). We chose a range of $10-130 \mu \mathrm{M}$ as this is the normal working range of most proteins. Interestingly we saw comparable labelling at $10 \mu \mathrm{M}$ to that of $130 \mu \mathrm{M}$, indicating this method can be used to label proteins at a range of concentrations. To assess labelling efficiency $50 \mu \mathrm{M}$ biotinylated trastuzumab Fab was incubated with 250 $\mu \mathrm{M}$ oxaziridine reagent. The biotinylated Fab reacted rapidly, achieving a maximum labelling efficiency of $80 \%$ (65\% single, $15 \%$ double labelled) after 1 hour (Fig. 2a). To test the stability of the resulting biotin sulfimide to hydrolysis, we incubated labelled Fab in PBS at $37^{\circ} \mathrm{C}$ for several days (Fig. 2b). Using the ReACT strategy, C-terminal methionine conjugates were $50 \%$ hydrolyzed within 3 days, whereas conjugates using the BioReACT approach were $100 \%$ stable 10 -days at $37^{\circ} \mathrm{C}$. This is comparable to the most stable mutants seen from an extensive methionine scan of the same Fab scaffold. ${ }^{9}$ These results demonstrated that BioReACT is an efficient method to rapidly and stably label proteins that contain the common Avi-Tag.
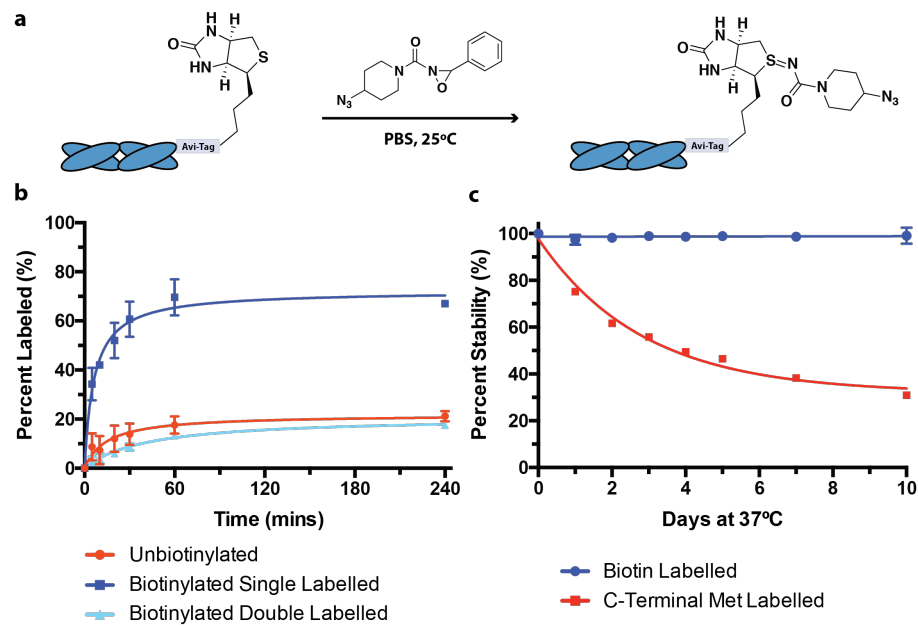

Figure 2: Biotin is a reactive handle for stable bioconjugation to proteins. a) Trastuzumab Fab was allowed to react with oxaziridine $\mathbf{2}$ in PBS for 1 hour at RT. b) Whole protein mass spectrometry was used to quantify labelling efficiency over time. Single labelling represents a singly biotinylated $\mathrm{Fab}$ with one oxaziridine adduct, while double labelling represents two oxaziridine adducts on a singly biotinylated Fab c) The singly labeled protein is $100 \%$ stable after incubation in $\mathrm{PBS}$ at $37^{\circ} \mathrm{C}$ for 10 days, whereas a C-terminal methionine oxaziridine conjugate is rapidly hydrolyzed. 
a
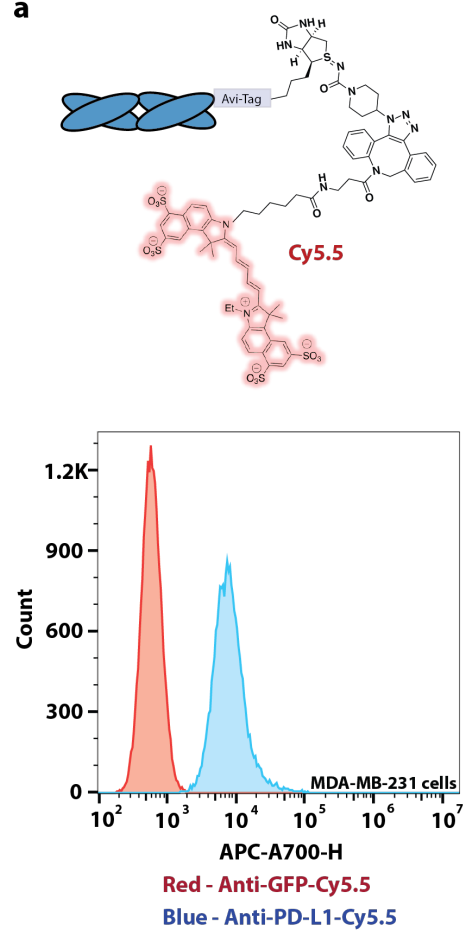
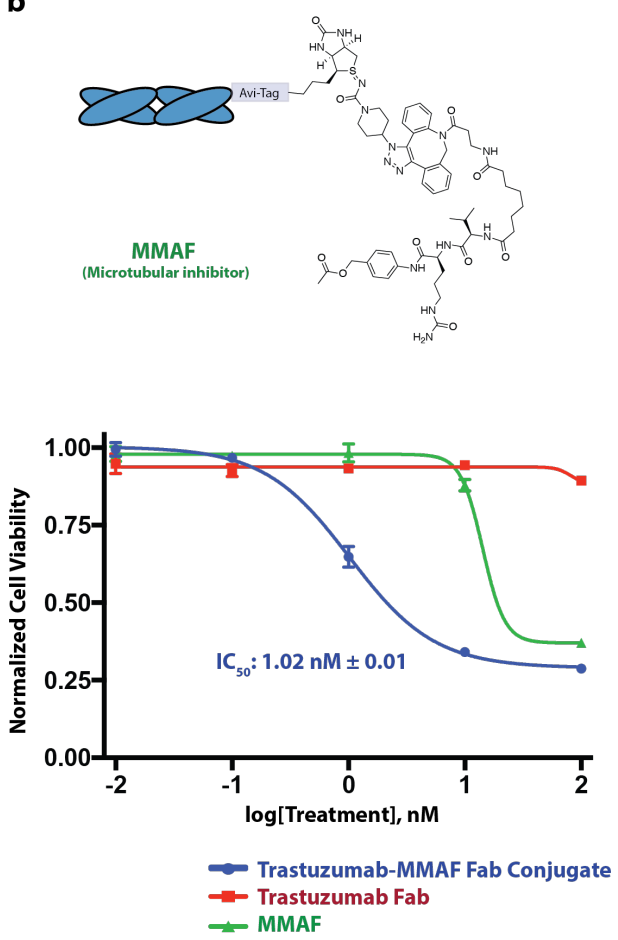

Figure 3. Biotinylated Fabs conjugated using BioReACT can be used to detect protein levels on cells and to form antibody drug conjugates. a) (Top) DBCO-Cy5.5 was reacted with azide labelled Anti-PD-L1 Fab to produce a fluorescently labelled Fab. (Bottom) Cy5.5 conjugated Fab detected PD-L1 by flow cytometry on the surface of MDA-MB-231 cells. b) (Top) DBCO-Val-Cit-PABA-MMAF was reacted with azide labelled trastuzumab Fab to form an antibody drug conjugate (ADC). (Bottom) The resulting ADC was incubated with SKBR3 cells for 3 days after which a cell titer glow assay was used to confirm dose-dependent killing.

To evaluate practical applications of BioReACT we generated flow cytometry and antibody-drug conjugate reagents. Flow cytometry is a method often used to evaluate protein levels on the surface of cells ${ }^{18}$. Most protocols involve multiple incubation steps, with either a primary antibody or a fluorophore-conjugated secondary antibody. We saw an opportunity to reduce the number of required incubations and wash steps by labelling biotinylated Fabs directly with a fluorescent dye in a controlled and site-specific manner. We first labelled biotinylated Fabs with oxaziridine 2, and the resulting azide-containing conjugate was allowed to react with a DBCO-Cy5.5 dye in a strain-promoted azide-alkyne cycloaddition. ${ }^{19,20}$ We labelled Fabs that target three different cell surface proteins, Her2, PDL1, and CDCP1. ${ }^{17,21,22}$ An anti-GFP Fab was fluorescently labelled as an isotype control. Each labelled Fab was able to detect its corresponding antigen on known cell lines (SKBR3 cell line for Her2 and MDA-MB-231 cells for PD-L1 and CDCP1, Fig. 3a, S4, S5). We next applied BioReACT to antibody drug conjugates (ADCs). Common methods to generate ADCs can result in a wide range of drug-to-antibody ratios (DARs). We anticipated BioReACT to give a well-defined DAR (assuming minimal reactivity with native cysteines) due to the predefined incorporation of a single biotin handle. We allowed azide-labelled trastuzumab Fab to react with DBCO-conjugated monomethyl auristatin F (MMAF), a cell-impermeable analog of MMAE. ${ }^{23}$ The resulting ADC selectively killed SKBR3 cells over $72 \mathrm{~h}$ more effectively than the warhead (MMAF) or the Fab alone (Fig. 3b). These results demonstrate that BioReACT is a useful tool for common applications that require proteinsmall molecule conjugation.
Due to the methionine dependence, to date the ReACT method has been constrained to labelling proteins. Biotinylation is very common in biological research, and we sought to exploit this to label other biomolecules. Oligonucleotide based therapeutics have gained traction as a way to modulate protein expression levels, and strategies for delivering these biomolecules involve their attachment to delivery vehicles such as carbohydrate polymers or peptides. ${ }^{3}$ These linkages often rely on bioconjugation strategies. We purchased a single stranded DNA oligonucleotide modified with a biotin tag at the 5' end and allowed it to react with a 5 -fold molar excess of oxaziridine 2 in water at $23{ }^{\circ} \mathrm{C}$ for one hour (Fig. 4). Oxaziridine 2 was dissolved in methanol to prevent subsequent signal suppression caused by either DMSO or DMF. MALDI-TOF Mass Spectrometry revealed highly efficient introduction of the azide handle onto DNA. In a parallel experiment, we showed that nonbiotinylated DNA does not label. Since biotin-conjugated oligonucleotides are cheap, stable, and readily available for purchase through commercial suppliers, BioReACT provides a rapid and practical method to generate stable small moleculeoligonucleotide conjugates.

The ReACT method has provided a way to selectively label methionine. Since methionine is rarely found on the surface of proteins, ReACT relies on the introduction of the amino acid by site-directed mutagenesis. Exhaustive screening is often required to find sites that readily react with oxaziridine reagents and provide hydrolytically stable conjugates, two properties that are critical for many bioconjugation applications. We have expanded the utility of these reagents by demonstrating their reactivity with biologically relevant and highly prevalent biotin. Biotin is commonly incorporated on commercially available surfaces, antibodies, proteins, oligonucleotides, and polymers in modern biological research. Additionally, it can be readily 
incorporated into proteins by a genetically encoded Avi-tag or by chemical conjugation. BioReACT works rapidly and efficiently with biotinylated proteins and DNA without the need to screen for a suitable site, and the resulting sulfimide is extremely stable to hydrolysis. While biotinylation is typically considered a synthetic endpoint, BioReACT enables rapid modification and customization through chemical conjugation.

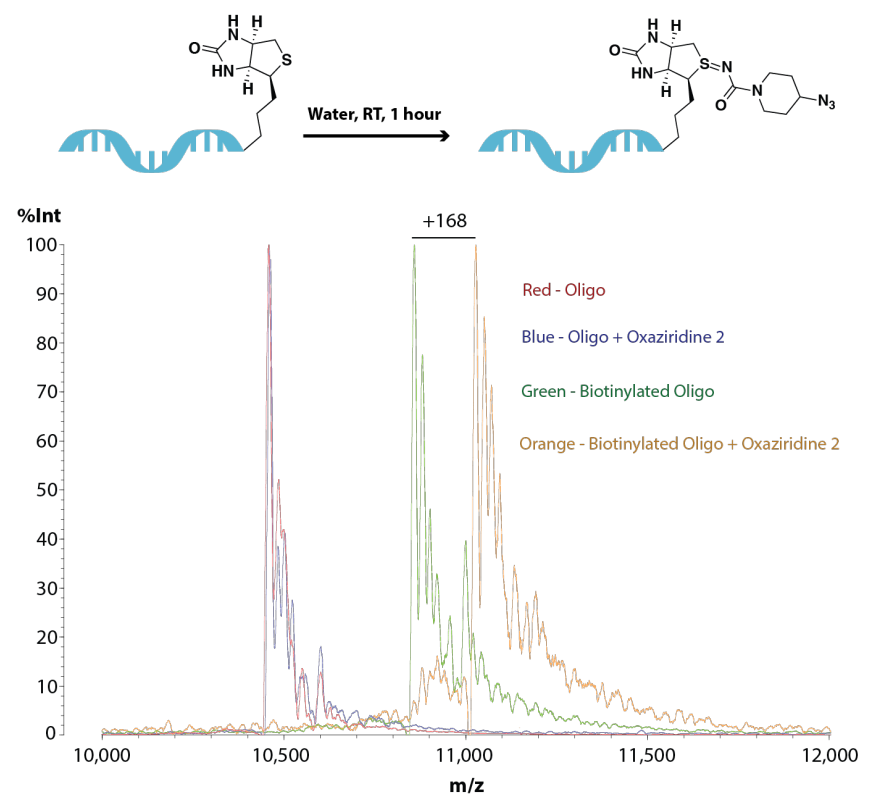

Figure 4: Expanding the applications of ReACT to label biotinylated DNA. (Top) Biotinylated DNA was exposed to 5 molar equivalents of oxaziridine 2 for 1 hour at RT in water to form the resulting sulfimide conjugate. (Bottom) MALDI-TOF Mass Spectrometry indicates efficient conjugation to biotinylated DNA (orange trace) and no labeling in the absence of biotin (blue trace).

\section{ACKNOWLEDGEMENTS:}

We thank the members of the Seiple and Wells laboratories for helpful discussion. S. Elledge for her generosity of general advice, plasmids, and protocols for oxaziridine labelling of Fabs. A. Tran for problem solving chemical synthesis protocols. This work was supported by the David and Lucille Packard Foundation.

\section{REFERENCES:}

(1) Beck, A.; Goetsch, L.; Dumontet, C.; Corvaïa, N. Strategies and Challenges for the next Generation of Antibody-Drug Conjugates. Nature Reviews Drug Discovery 2017, 16 (5), 315-337. https://doi.org/10.1038/nrd.2016.268.

(2) Ohata, J.; Krishnamoorthy, L.; Gonzalez, M. A.; Xiao, T.; Iovan, D. A.; Toste, F. D.; Miller, E. W.; Chang, C. J. An ActivityBased Methionine Bioconjugation Approach To Developing Proximity-Activated Imaging Reporters. ACS Cent. Sci. 2020, 6 (1), 32-40. https://doi.org/10.1021/acscentsci.9b01038.

(3) Benizri, S.; Gissot, A.; Martin, A.; Vialet, B.; Grinstaff, M. W.; Barthélémy, P. Bioconjugated Oligonucleotides: Recent Developments and Therapeutic Applications. Bioconjugate Chem. 2019, 30 (2), 366-383. https://doi.org/10.1021/acs.bioconjchem.8b00761. (4) Spicer, C. D.; Davis, B. G. Selective Chemical Protein Modification. Nat Commun 2014, 5 (1), 4740.

https://doi.org/10.1038/ncomms5740.

(5) Taylor, M. T.; Nelson, J. E.; Suero, M. G.; Gaunt, M. J. A Protein Functionalization Platform Based on Selective Reactions at Methionine Residues. Nature 2018, 562 (7728), 563-568. https://doi.org/10.1038/s41586-018-0608-y.
(6) Lin, S.; Yang, X.; Jia, S.; Weeks, A. M.; Hornsby, M.; Lee, P. S.; Nichiporuk, R. V.; Iavarone, A. T.; Wells, J. A.; Toste, F. D.; Chang, C. J. Redox-Based Reagents for Chemoselective Methionine Bioconjugation. Science 2017, 355 (6325), 597-602.

https://doi.org/10.1126/science.aal3316.

(7) Dyer, K. F. The Quiet Revolution: A New Synthesis of Biological Knowledge. Journal of Biological Education 1971, 5 (1), 1524. https://doi.org/10.1080/00219266.1971.9653663.

(8) Shaytan, A. K.; Shaitan, K. V.; Khokhlov, A. R. Solvent Accessible Surface Area of Amino Acid Residues in Globular Proteins: Correlation of Apparent Transfer Free Energies with Experimental Hydrophobicity Scales. Biomacromolecules 2009, 10 (5), 1224-1237. https://doi.org/10.1021/bm8015169.

(9) Elledge, S. K.; Tran, H. L.; Christian, A. H.; Steri, V.; Hann, B.; Toste, F. D.; Chang, C. J.; Wells, J. A. Systematic Identification of Engineered Methionines and Oxaziridines for Efficient, Stable, and Site-Specific Antibody Bioconjugation. Proc Natl Acad Sci USA 2020, 117 (11), 5733-5740.

https://doi.org/10.1073/pnas.1920561117.

(10) Christian, A. H.; Jia, S.; Cao, W.; Zhang, P.; Meza, A. T.; Sigman, M. S.; Chang, C. J.; Toste, F. D. A Physical Organic Approach to Tuning Reagents for Selective and Stable Methionine Bioconjugation. J. Am. Chem. Soc. 2019, 141 (32), 12657-12662. https://doi.org/10.1021/jacs.9b04744.

(11) Sung, K.; Maloney, M. T.; Yang, J.; Wu, C. A Novel Method for Producing Mono-Biotinylated, Biologically Active Neurotrophic Factors: An Essential Reagent for Single Molecule Study of Axonal Transport. J Neurosci Methods 2011, 200 (2), 121-128. https://doi.org/10.1016/j.jneumeth.2011.06.020.

(12) Williams, J. G. K.; Steffens, D. L.; Anderson, J. P.; Urlacher, T. M.; Lamb, D. T.; Grone, D. L.; Egelhoff, J. C. An Artificial Processivity Clamp Made with Streptavidin Facilitates Oriented Attachment of Polymerase-DNA Complexes to Surfaces. Nucleic Acids Res 2008, 36 (18), e121. https://doi.org/10.1093/nar/gkn531.

(13) Valadon, P.; Darsow, B.; Buss, T. N.; Czarny, M.; Griffin, N. M.; Nguyen, H. N.; Oh, P.; Borgstrom, P.; Chrastina, A.;

Schnitzer, J. E. Designed Auto-Assembly of Nanostreptabodies for Rapid Tissue-Specific Targeting in Vivo. J Biol Chem 2010, 285 (1), 713-722. https://doi.org/10.1074/jbc.M109.061838.

(14) Sims, S.; Willberg, C.; Klenerman, P. MHC-Peptide Tetramers for the Analysis of Antigen-Specific T Cells. Expert Rev Vaccines 2010, 9 (7), 765-774. https://doi.org/10.1586/erv.10.66.

(15) Livnah, O.; BAYERt, E. A.; WILCHEKt, M.; Sussman, J. L. Three-Dimensional Structures of Avidin and the Avidin- Biotin Complex. Proc. Natl. Acad. Sci. USA 1993, 5.

(16) Glasel, J. A. A Nuclear Magnetic Resonance Investigation of Biotin. The Biotin Sulfonium Ion *. Biochemistry 1966, 5 (6), 1851-1855. https://doi.org/10.1021/bi00870a010.

(17) Cho, H.-S.; Mason, K.; Ramyar, K. X.; Stanley, A. M.; Gabelli, S. B.; Jr, D. W. D.; Leahy, D. J. Structure of the Extracellular Region of HER2 Alone and in Complex with the Herceptin Fab. 2003, 421,5 .

(18) McKinnon, K. M. Flow Cytometry: An Overview. Curr Protoc Immunol 2018, 120, 5.1.1-5.1.11.

https://doi.org/10.1002/cpim.40.

(19) Agard, N. J.; Prescher, J. A.; Bertozzi, C. R. A Strain-Promoted [3 + 2] Azide-Alkyne Cycloaddition for Covalent Modification of Biomolecules in Living Systems. J. Am. Chem. Soc. 2004, 126 (46), 15046-15047. https://doi.org/10.1021/ja044996f.

(20) Dommerholt, J.; Rutjes, F. P. J. T.; van Delft, F. L. StrainPromoted 1,3-Dipolar Cycloaddition of Cycloalkynes and Organic Azides. Top Curr Chem (Z) 2016, 374 (2), 16.

https://doi.org/10.1007/s41061-016-0016-4.

(21) Zhang, F.; Qi, X.; Wang, X.; Wei, D.; Wu, J.; Feng, L.; Cai, H.; Wang, Y.; Zeng, N.; Xu, T.; Zhou, A.; Zheng, Y. Structural Basis of the Therapeutic Anti-PD-L1 Antibody Atezolizumab. Oncotarget 2017, 8 (52), 90215-90224. https://doi.org/10.18632/oncotarget.21652.

(22) Martinko, A. J.; Truillet, C.; Julien, O.; Diaz, J. E.; Horlbeck, M. A.; Whiteley, G.; Blonder, J.; Weissman, J. S.; Bandyopadhyay, S.; Evans, M. J.; Wells, J. A. Targeting RAS-Driven Human Cancer Cells with Antibodies to Upregulated and Essential Cell- 
Surface Proteins. eLife 2018, 7, e31098. https://doi.org/10.7554/eLife.31098.

(23) Doronina, S. O.; Mendelsohn, B. A.; Bovee, T. D.; Cerveny, C. G.; Alley, S. C.; Meyer, D. L.; Oflazoglu, E.; Toki, B. E.;

Sanderson, R. J.; Zabinski, R. F.; Wahl, A. F.; Senter, P. D. Enhanced Activity of Monomethylauristatin F through Monoclonal Antibody Delivery: Effects of Linker Technology on Efficacy and Toxicity. Bioconjugate Chem. 2006, 17 (1), 114-124.

https://doi.org/10.1021/bc0502917.
(24) Hornsby, M.; Paduch, M.; Miersch, S.; Sääf, A.; Matsuguchi, T.; Lee, B.; Wypisniak, K.; Doak, A.; King, D.; Usatyuk, S.; Perry, K.; Lu, V.; Thomas, W.; Luke, J.; Goodman, J.; Hoey, R. J.; Lai, D.; Griffin, C.; Li, Z.; Vizeacoumar, F. J.; Dong, D.; Campbell, E.; Anderson, S.; Zhong, N.; Gräslund, S.; Koide, S.; Moffat, J.; Sidhu, S.; Kossiakoff, A.; Wells, J. A High Through-Put Platform for Recombinant Antibodies to Folded Proteins. Mol Cell Proteomics 2015, 14 (10), 2833-2847. https://doi.org/10.1074/mcp.O115.052209. 